\title{
Clinical Benefit of Valvular Surgery in Patients with Chronic Kidney Disease
}

\author{
Yan Chen, ${ }^{1,2}$ MD, Wing-Kuk Au, ${ }^{3}$ MBBS, Daniel Chan, ${ }^{3}$ MBBS, Ko-Yung Sit, ${ }^{3}$ MBBS, Zhe Zhen, ${ }^{1}$ MD, \\ Kar-Lai Ho, ${ }^{3}$ MBBS, Debbie Wong, ${ }^{1}$ BSc, Lai-Ming Ho, ${ }^{4} \mathrm{PhD}$, Desmond Yap, ${ }^{5} \mathrm{MD}$, \\ Yui-Ming Lam, ${ }^{1}$ MBBS, Chu-Pak Lau, ${ }^{1} \mathrm{MD}$, Hung-Fat Tse, ${ }^{1}$ MD, \\ Tak-Mao Chan, ${ }^{5}$ MD and Kai-Hang Yiu, ${ }^{1,2}$ MD
}

\begin{abstract}
Summary
Concomitant chronic kidney disease (CKD) is common in patients with significant valvular heart disease (VHD). This study sought to evaluate the clinical benefit of valvular surgery in patients with concomitant CKD.

We evaluated 349 patients with significant VHD who were referred for surgery. Patients were divided into those with CKD stage $\geq 3$ (CKD patients; $n=88$ ) and those with CKD stage 1 or 2 (no CKD patients; $n=$ 261). 63 patients did not receive surgery, of which 20 patients had CKD and 43 had no CKD. Mortality and change in eGFR were assessed after a median follow-up of 21 months.

In the whole study population, $25 \%$ of the patients had CKD and these patients had higher mortality than those with no CKD. The annual mortality rates of patients with CKD who did and did not undergo surgery were $7.9 \%$ and $28.0 \%$, respectively. In patients with no CKD, the annual mortality rates of those who did and did not undergo surgery were $1.8 \%$ and $2.3 \%$, respectively. Importantly, surgery was associated with significant survival benefit in patients with CKD (log-rank test, $P<0.01$ ), but was neutral in patients with no CKD. Multivariable analysis confirmed the survival benefit of valvular surgery in all patients, which was most significant in patients with CKD. Furthermore, eGFR was preserved in patients who underwent valvular surgery but declined significantly in those who did not.
\end{abstract}

CKD is common in patients with significant VHD and, if left untreated surgically, these patients exhibit a high mortality.

(Int Heart J 2018; 59: 759-765)

Key words: Renal dysfunction, Valvular heart disease, Estimated glomerular filtration rate, Outcome

$\mathrm{V}$ alvular heart disease (VHD) constitutes a special population in cardiovascular medicine and is subject of growing concern and focus because of its increased prevalence due to the aging population. ${ }^{1)}$ Unlike heart failure and coronary artery disease, where medication is the mainstay of successful treatment, patients with significant VHD often require corrective valvular surgery to optimize long-term survival. ${ }^{2,3)}$ However, the decision between continued medical management and valvular surgery in patients with comorbidities is challenging for both patients and physicians. One of the determining factors is the presence of chronic kidney disease (CKD), likewise a rapidly growing health problem due to the aging population, ${ }^{4)}$ that is closely associated with morbidity and mortality following valvular surgery. ${ }^{5,6)}$ As a result, a significant number of patients have been denied surgery as they were considered too high risk due to concomitant CKD.
Although valvular surgery is associated with a dismal post-operative outcome, its long-term clinical outcome compared to that of continued medical therapy in patients with significant CKD has not been studied. The aim of the present study was thus to evaluate the clinical benefit of valvular surgery in patients with concomitant significant CKD.

\section{Method}

Study population: From January 2012 to February 2015, 414 consecutive patients with significant VHD for whom conventional surgical correction was indicated, including those with significant clinical symptoms, dilated left ventricular dimension, and/or impaired left ventricular function, were prospectively recruited. ${ }^{3)}$ Patients with a documented history of congenital heart disease $(n=10)$, acute

From the 'Division of Cardiology, Department of Medicine, The University of Hong Kong, Queen Mary Hospital, Hong Kong, China, ${ }^{2}$ Division of Cardiology, Department of Medicine, The University of Hong Kong Shenzhen Hospital, Shenzhen, China, ${ }^{3}$ Department of Surgery, The University of Hong Kong, Queen Mary Hospital, Hong Kong, China, ${ }^{4}$ School of Public Health, University of Hong Kong, Hong Kong, China and ${ }^{5}$ Divsion of Nephrology, Department of Medicine, The University of Hong Kong, Queen Mary Hospital, Hong Kong, China.

Address for correspondence: Kai-Hang Yiu, MD, Division of Cardiology, Department of Medicine, The University of Hong Kong, Queen Mary Hospital, Room 1929B, Block K, Hong Kong, China. E-mail: khkyiu@ @ku.hk

Received for publication August 9, 2017. Revised and accepted October 3, 2017.

Released in advance online on J-STAGE June 20, 2018.

doi: 10.1536/ihj.17-460

All rights reserved by the International Heart Journal Association. 
infective endocarditis $(n=4)$, underlying renal parenchymal disease $(n=23)$, end-stage renal disease requiring dialysis $(n=8)$, or who refused to participate $(n=20)$ were excluded. A total of 349 patients suitable for final analysis were included. Indications for surgery in patients with VHD were evaluated according to the combined guidelines. ${ }^{2,3)}$ The predominant left heart valvular lesion indicated for surgery was categorized as either single or mixed (defined as more than one valvular lesion with at least a moderate degree of severity). Concomitant tricuspid annuloplasty and coronary artery bypass graft during left heart valvular surgery was documented. The study was part of the Chinese Valvular Heart Disease Study to evaluate the pattern of disease, pathophysiology, and clinical outcome in Chinese patients with VHD. ${ }^{7}$ The study was approved by the ethics committee of the West Cluster Hospital Authority of Hong Kong, and all subjects gave written informed consent.

Estimated glomerular filtration rate (eGFR): The serum creatinine level within 1 week of the echocardiography examination or surgery, whichever was closer, was used to calculate the baseline eGFR using the Modified Diet in Renal Disease Equation. ${ }^{8)}$ Two categories of eGFR based on the National Kidney Foundation recommendations were compared: CKD stage 1 or 2 (eGFR $\geq 60 \mathrm{~mL} /$ minute $\left./ 1.73 \mathrm{~m}^{2}\right)$, classified as no CKD; CKD stage $\geq 3$ $\left(\mathrm{eGFR}<60 \mathrm{~mL} /\right.$ minute $\left./ 1.73 \mathrm{~m}^{2}\right)$, classified as CKD according to prior studies. ${ }^{9-12)}$

Clinical parameters: Clinical data on preoperative variables were collected from patient records by one investigator. The etiology of VHD was recorded as chronic rheumatic heart disease (CRHD), bicuspid aortic valve, mitral valve prolapse, and degenerative valvular disease according to the predominant lesion of the valve. The New York Heart Association (NYHA) classification was recorded as class I/II or III/IV, and the status of atrial fibrillation (AF) was also recorded for each subject. Conventional cardiovascular risk factors such as history of diabetes mellitus, hyperlipidemia, hypertension, and smoking status were documented. Data on prescription of angiotensinconverting enzyme inhibitor (ACEI) or angiotensin II receptor blocker (ARB), beta blocker, calcium channel blocker, and statin were retrieved from the Hospital Authority records.

Clinical follow-up: All-cause mortality was retrieved from the inter-hospital computer medical system. Followup eGFR was calculated using the creatinine level measured at least 6 months after the baseline eGFR or that available at the time of death. Significant CKD progression was defined as a $30 \%$ reduction in eGFR from the baseline value. ${ }^{13)}$ The annual rate of decline of eGFR (slope of eGFR over time) as a measure of CKD was also examined.

Conventional echocardiography: Detailed transthoracic echocardiography was performed in all subjects. Patients were imaged in the left lateral decubitus position using a commercially available echocardiography system (Vingmed E9, General Electric Vingmed Ultrasound; Milwaukee, WI, USA). A 3.5-MHz transducer was used to obtain images that were digitally stored in cine-loop format (5 cardiac cycles). Offline analysis was performed using
EchoPAC version 112.0 (General Electric Vingmed, Horten, Norway). The left ventricular (LV) systolic and diastolic volumes and ejection fraction were measured according to the modified biplane Simpson's rule. ${ }^{14)}$

Statistical analysis: Continuous variables are expressed as mean \pm standard deviation and were compared with the independent sample $t$-test. Categorical variables are reported as frequencies or proportions and were compared using the chi-squared or the Fisher's exact test if at least one cell had an expected cell count below 5 .

A Kaplan-Meier curve was constructed and the percentage survival among patients with and without CKD and in those with and without valvular surgery were compared using the log-rank test. A Cox regression survival model adjusted for age, sex, diabetes mellitus, hypertension, hyperlipidemia, smoking, AF, ACEI/ARB use, and baseline eGFR was performed to evaluate the impact of valvular surgery on mortality. Logistic and linear regressions were used to assess the association of valvular surgery with CKD progression and annual rate of eGFR decline after adjusting for covariates. All statistical analyses were performed using the statistical package SPSS for windows (Version 20.0; SPSS, Chicago, IL, USA) and the $P$ values reported are two-sided for consistency. A $P$ value $<0.05$ was considered statistically significant.

\section{Results}

Clinical demographics and prevalence of renal dysfunction: The mean age of the whole study population was 64 years and $56 \%$ were female (Table I). Nearly $70 \%$ of the patients had AF and over $60 \%$ had CRHD as the cause of valve pathology. The most common single valvular lesion was mitral regurgitation and $43.8 \%$ had mixed valvular disease. The mean eGFR of the whole population was $71.7 \mathrm{~mL} /$ minute $/ 1.73 \mathrm{~m}^{2}, 88$ patients $(25 \%)$ had CKD (CKD stage $\geq 3$ ) and 261 patients had no CKD (CKD stage 1 or 2). Patients with CKD were older and had a higher prevalence of diabetes mellitus and hypertension than those with no CKD. Nonetheless, the sex, NYHA class III/IV, etiology of VHD, and echocardiography parameters were similar between the two groups. For the whole study population, the prevalence of CKD increased with age and was present in up to $30 \%$ of the patients aged above 60 years (Figure 1), but was similar for males and females $(23.8 \%$ versus $26.3 \%, P=0.62)$.

Clinical characteristic of patients with and without valvular surgery: In the whole study population, 286 patients underwent valvular surgery and 63 did not received surgery, of which 20 patients had CKD and 43 had no CKD. The reasons for not performing valvular surgery despite a positive indication at the time of assessment are as follows: declined by patient because of considering too high risk $(n=33)$ and those pending surgery $(n=30)$. For the patients who underwent surgery, the following valvular surgery was performed: mitral valve replacement ( $n$ $=74)$, mitral valve repair $(n=76)$, aortic valve replacement $(n=45)$, and dual valvular replacement $(n=91)$. Furthermore, all of them received cardiopulmonary bypass, $171(59.8 \%)$ had concomitant tricuspid annuloplasty, and $20(5.7 \%)$ received concomitant coronary artery by- 
Table I. Clinical Characteristics of Patients

\begin{tabular}{|c|c|c|c|c|}
\hline Variables & $\begin{array}{c}\text { Total } \\
(n=349)\end{array}$ & $\begin{array}{c}\text { CKD stage } \geq 3 \\
(n=88)\end{array}$ & $\begin{array}{c}\text { CKD stage } 1 \text { or } 2 \\
(n=261)\end{array}$ & $P$ \\
\hline \multicolumn{5}{|l|}{ Clinical demographics } \\
\hline Age, years & $64.4 \pm 10.1$ & $68.5 \pm 8.0$ & $63.0 \pm 10.4$ & $<0.01$ \\
\hline Male, $n(\%)$ & $151(43.3)$ & $36(40.9)$ & $115(44.1)$ & 0.61 \\
\hline Diabetes mellitus, $n(\%)$ & $61(17.5)$ & $23(26.1)$ & $38(14.6)$ & 0.01 \\
\hline Hypertension, $n(\%)$ & $72(20.6)$ & $27(30.7)$ & $45(17.2)$ & $<0.01$ \\
\hline Hyperlipidemia, $n(\%)$ & $61(17.5)$ & $20(22.7)$ & $41(15.7)$ & 0.13 \\
\hline Smoking, $n(\%)$ & $58(16.6)$ & $17(19.3)$ & $41(15.7)$ & 0.43 \\
\hline Atrial fibrillation, $n(\%)$ & $243(69.6)$ & $64(72.7)$ & $179(68.6)$ & 0.47 \\
\hline NYHA class III/IV, $n(\%)$ & $86(24.6)$ & $26(29.5)$ & $60(23.0)$ & 0.22 \\
\hline \multicolumn{5}{|l|}{ Etiology of valvular heart disease, $n(\%)$} \\
\hline CRHD & $210(60.2)$ & $51(58.0)$ & $159(60.9)$ & 0.43 \\
\hline Bicuspid aortic valve & $7(2.0)$ & $1(1.1)$ & $6(2.3)$ & \\
\hline Mitral valve prolapse & $86(24.6)$ & $27(30.7)$ & $59(22.6)$ & \\
\hline Degenerative valve disease & $46(13.2)$ & $9(10.2)$ & $37(14.2)$ & \\
\hline \multicolumn{5}{|l|}{ Left sided valvular lesion, $n(\%)$} \\
\hline \multicolumn{5}{|l|}{ Single $(n=196)$} \\
\hline Mitral stenosis & $33(16.8)$ & $8(18.2)$ & $25(16.4)$ & 0.64 \\
\hline Mitral regurgitation & $113(57.7)$ & $28(63.6)$ & $85(55.9)$ & \\
\hline Aortic stenosis & $29(14.8)$ & $4(9.1)$ & $25(16.4)$ & \\
\hline Aortic regurgitation & $21(10.7)$ & $4(9.1)$ & $17(11.2)$ & \\
\hline Mixed $(n=153)$ & $153(43.8)$ & $44(50.0)$ & $109(41.8)$ & 0.18 \\
\hline \multicolumn{5}{|l|}{ Predominant surgical details $(n=268), n(\%)$} \\
\hline Mitral valve repair & $76(26.6)$ & $26(38.2)$ & $50(22.9)$ & 0.08 \\
\hline Mitral valve replacement & $74(25.9)$ & $15(22.1)$ & $59(27.1)$ & \\
\hline Aortic valve replacement & $45(15.7)$ & $7(10.3)$ & $38(17.4)$ & \\
\hline Dual valvular replacement & $91(31.8)$ & $20(29.4)$ & $71(32.6)$ & \\
\hline Combined tricuspid annuloplasty, $n(\%)$ & $171(59.8)$ & $47(69.1)$ & $124(56.9)$ & 0.07 \\
\hline \multicolumn{5}{|l|}{ Renal function } \\
\hline eGFR (mL/minute/1.73 m²) & $71.7 \pm 18.3$ & $49.8 \pm 8.1$ & $79.0 \pm 14.5$ & $<0.01$ \\
\hline \multicolumn{5}{|l|}{ Echocardiography parameters } \\
\hline LVEDV (mL) & $102.3 \pm 49.6$ & $102.5 \pm 48.2$ & $102.2 \pm 50.2$ & 0.97 \\
\hline LVESV (mL) & $41.3 \pm 27.2$ & $42.7 \pm 31.6$ & $40.9 \pm 25.6$ & 0.59 \\
\hline $\operatorname{LVEF}(\%)$ & $60.6 \pm 9.2$ & $60.1 \pm 10.0$ & $60.8 \pm 8.9$ & 0.54 \\
\hline \multicolumn{5}{|l|}{ Drugs, $n(\%)$} \\
\hline ACEI/ARB & $177(50.7)$ & $48(54.5)$ & $129(49.4)$ & 0.41 \\
\hline Calcium channel blocker & $90(25.8)$ & $20(22.7)$ & $70(26.8)$ & 0.45 \\
\hline Beta blocker & $187(53.6)$ & $49(55.7)$ & $138(52.9)$ & 0.65 \\
\hline Statin & $122(35.0)$ & $95(36.4)$ & $27(30.7)$ & 0.33 \\
\hline
\end{tabular}

Values are mean $\pm \mathrm{SD}$ or $n(\%)$. ACEI indicates angiotensin converting enzyme inhibitor; ARB, angiotensin receptor blocker; CKD, chronic kidney disease; CRHD, chronic rheumatic heart disease; eGFR, estimated glomerular filtration rate; LVEDV, left ventricular end diastolic volume; LVEF, LV ejection fraction; LVESV, LV end systolic volume; and NYHA, New York Heart Association.

pass graft. The predominant surgical types were no difference between patients with and without CKD (Table I). For patients with CKD, those who did not receive surgery were more likely to have mixed valvular disease (Table II). For patients with no CKD, those who did not receive surgery were more likely to be older, have single valvular disease, a smaller LV dimension, and less AF compared to those who received surgery.

Clinical outcome: The median follow-up was 21 months (range 1-44 months) and death occurred in 26 patients: all were due to a cardiovascular cause (24 heart failurerelated and two sudden cardiac deaths). The annual mortality rates for patients with and with no valvular surgery were $3.3 \%$ and $8.1 \%$, respectively.

The Kaplan-Meier survival curves comparing the prognostic value of valvular surgery in patients with and with no CKD are shown in Figure 2. The annual mortality rates of CKD patients with and with no valvular surgery were $7.9 \%$ and $28.0 \%$, respectively. In patients with no $\mathrm{CKD}$, the annual mortality rates of those with and with no surgery were $1.8 \%$ and $2.3 \%$, respectively. In the whole study population, patients with CKD had a higher mortality than those with no CKD (Figure $2 \mathrm{~A}, P<0.01$ ). Importantly, surgery was associated with a significant survival benefit in patients with CKD (solid line, Figure 2B) but was neutral in patients with no CKD (dotted line, Figure $2 \mathrm{~B}$ ). Furthermore, patients with CKD who did not undergo surgery had the worst prognosis among all subgroups (Figure 2B). Among the 33 patients who declined surgery, mortality occurred in $4 / 10(40 \%)$ patients who had CKD compared to only $2 / 23(8.7 \%)$ patients with no CKD $(P=0.05)$. Similarly, among the 30 patients who 


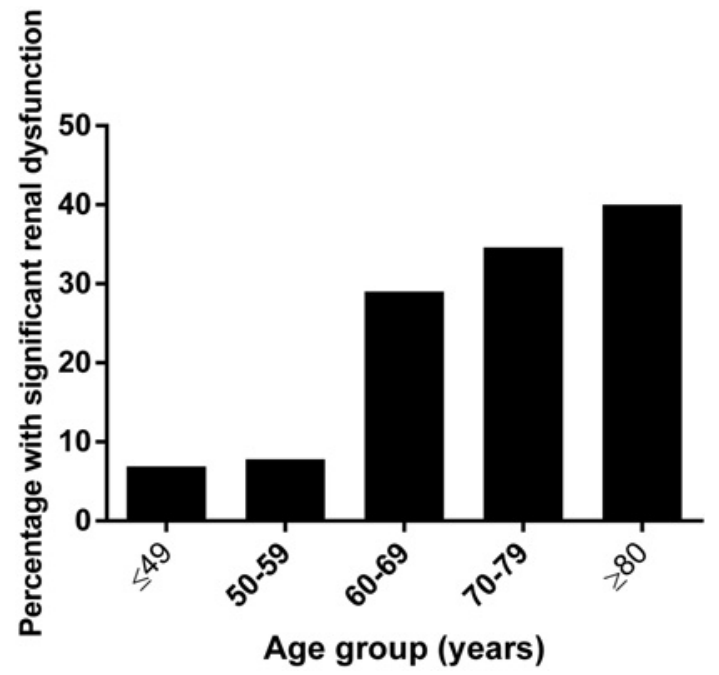

Figure 1. Percentage of patients with significant renal dysfunction defined as chronic kidney disease (CKD) stage $\geq 3$ according to different age groups. were pending surgery, 3/10 (30\%) with CKD died during the waiting period (median 2 months, range 1-3 months) compared to none of those with no CKD $(P=0.03)$. Multivariable Cox regression confirmed the survival benefit of valvular surgery in all patients (Table III). Importantly, the adjusted survival benefit was most significant for patients with CKD but neutral for those with no CKD.

CKD progression in patients with and without valvular surgery: In the whole population, the mean eGFR declined from $71.7 \pm 18.3$ to $70.3 \pm 22.8 \mathrm{~mL} /$ minute $/ 1.73$ $\mathrm{m}^{2}$. The eGFR was preserved in patients who underwent valvular surgery $(72.4 \pm 18.6$ to $72.1 \pm 23.1 \mathrm{~mL} /$ minute/ $1.73 \mathrm{~m}^{2}, P=0.78$ ), but declined significantly in those who did not $(68.4 \pm 16.7$ to $62.1 \pm 19.6 \mathrm{~mL} /$ minute $/ 1.73$ $\left.\mathrm{m}^{2}, P<0.01\right)$. Comparison with those who underwent valvular surgery revealed that the distribution of eGFR percentage change in patients without surgery was more skewed towards a negative value, indicating a more prevalent decline in eGFR (Figure 3). Furthermore, the incidence of $>30 \%$ eGFR loss and the annual rate of eGFR decline was greater in patients with no surgery than in those who underwent valvular surgery, even after multivariable adjustment (Table IV).

Table II. Clinical Characteristics of Patients with and without Valvular Surgery

\begin{tabular}{|c|c|c|c|c|c|c|}
\hline & \multicolumn{3}{|c|}{ CKD stage $\geq 3(n=88)$} & \multicolumn{3}{|c|}{ CKD stage 1 or $2(n=261)$} \\
\hline & $\begin{array}{l}\text { Surgery } \\
(n=68)\end{array}$ & $\begin{array}{l}\text { No surgery } \\
\quad(n=20)\end{array}$ & $P$ & $\begin{array}{c}\text { Surgery } \\
(n=218)\end{array}$ & $\begin{array}{l}\text { No surgery } \\
\quad(n=43)\end{array}$ & $P$ \\
\hline Age, years & $68.3 \pm 6.9$ & $69.2 \pm 11.0$ & 0.74 & $62.1 \pm 10.1$ & $67.2 \pm 10.6$ & $<0.01$ \\
\hline Male, $n(\%)$ & $27(39.7)$ & $9(45.0)$ & 0.67 & $93(42.7)$ & $22(51.2)$ & 0.31 \\
\hline Diabetes mellitus, $n(\%)$ & $17(25.0)$ & $6(30.0)$ & 0.66 & $33(15.1)$ & $5(11.6)$ & 0.55 \\
\hline Hypertension, $n(\%)$ & $20(29.4)$ & $7(35.0)$ & 0.63 & $38(17.4)$ & $7(16.3)$ & 0.86 \\
\hline Hyperlipidemia, $n(\%)$ & $16(23.5)$ & $4(20.0)$ & 0.74 & $35(16.1)$ & $6(14.0)$ & 0.73 \\
\hline Smoking, $n(\%)$ & $12(17.6)$ & $5(25.0)$ & 0.52 & $37(17.0)$ & $4(9.3)$ & 0.21 \\
\hline Atrial fibrillation, $n(\%)$ & $48(70.6)$ & $16(80.0)$ & 0.41 & $156(71.6)$ & $23(53.5)$ & 0.02 \\
\hline NYHA class III/IV, $n(\%)$ & $20(29.4)$ & $6(30.0)$ & 0.96 & $55(25.2)$ & $5(11.6)$ & 0.06 \\
\hline \multicolumn{7}{|l|}{ Etiology of valvular heart disease, $n(\%)$} \\
\hline CRHD & $39(57.4)$ & $12(60.0)$ & 0.73 & $139(63.8)$ & $20(46.5)$ & 0.08 \\
\hline Bicuspid aortic valve & $1(1.4)$ & $0(0)$ & & $6(2.8)$ & $0(0)$ & \\
\hline Mitral valve prolapse & $22(32.4)$ & $5(25.0)$ & & $45(20.6)$ & $14(32.6)$ & \\
\hline Degenerative valve disease & $6(8.8)$ & $3(15.0)$ & & $28(12.8)$ & $9(20.9)$ & \\
\hline \multicolumn{7}{|l|}{ Left sided valvular lesion, $n(\%)$} \\
\hline \multicolumn{7}{|l|}{ Single } \\
\hline Mitral stenosis & $7(17.9)$ & $1(20.0)$ & 0.74 & $21(17.4)$ & $4(12.9)$ & 0.28 \\
\hline Mitral regurgitation & $24(61.5)$ & $4(80.0)$ & & $64(52.9)$ & $21(67.7)$ & \\
\hline Aortic stenosis & $4(10.3)$ & $0(0)$ & & $23(19.0)$ & $2(6.5)$ & \\
\hline Aortic regurgitation & $4(10.3)$ & $0(0)$ & & $13(10.7)$ & $4(12.9)$ & \\
\hline Mixed & $29(42.6)$ & $15(75.0)$ & 0.01 & $97(44.5)$ & $12(27.9)$ & 0.04 \\
\hline \multicolumn{7}{|l|}{ Predominant surgical details, $n(\%)$} \\
\hline Mitral valve repair & $26(38.2)$ & - & - & $50(22.9)$ & - & - \\
\hline Mitral valve replacement & $15(22.1)$ & - & - & $59(27.1)$ & - & - \\
\hline Aortic valve replacement & $7(10.3)$ & - & - & $38(17.4)$ & - & - \\
\hline Dual valvular replacement & $20(29.4)$ & - & - & $71(32.6)$ & - & - \\
\hline Combined tricuspid annuloplasty, $n(\%)$ & $47(69.1)$ & - & - & $124(56.9)$ & - & - \\
\hline LVEDV $(\mathrm{mL})$ & $102.3 \pm 46.7$ & $103.5 \pm 54.2$ & 0.92 & $104.4 \pm 53.0$ & $91.5 \pm 30.4$ & 0.03 \\
\hline LVESV (mL) & $43.3 \pm 29.9$ & $40.7 \pm 37.8$ & 0.75 & $42.3 \pm 27.2$ & $33.6 \pm 13.0$ & $<0.01$ \\
\hline $\operatorname{LVEF}(\%)$ & $59.4 \pm 9.9$ & $62.5 \pm 10.4$ & 0.24 & $60.3 \pm 9.2$ & $63.1 \pm 7.0$ & 0.06 \\
\hline ACEI/ARB & $39(57.4)$ & $9(45.0)$ & 0.33 & $106(48.6)$ & $23(53.5)$ & 0.56 \\
\hline Calcium channel blocker, $n(\%)$ & $15(22.1)$ & $5(25.0)$ & 0.77 & $57(26.1)$ & $13(30.2)$ & 0.58 \\
\hline Beta blocker, $n(\%)$ & $40(58.8)$ & $9(45.0)$ & 0.27 & $116(53.2)$ & $22(51.2)$ & 0.81 \\
\hline Statin, $n(\%)$ & $21(30.9)$ & $6(30.0)$ & 0.94 & $80(36.7)$ & $15(34.9)$ & 0.82 \\
\hline
\end{tabular}

Values are mean $\pm \mathrm{SD}$ or $n(\%)$. Abbreviations similar to those of Table I. 
A

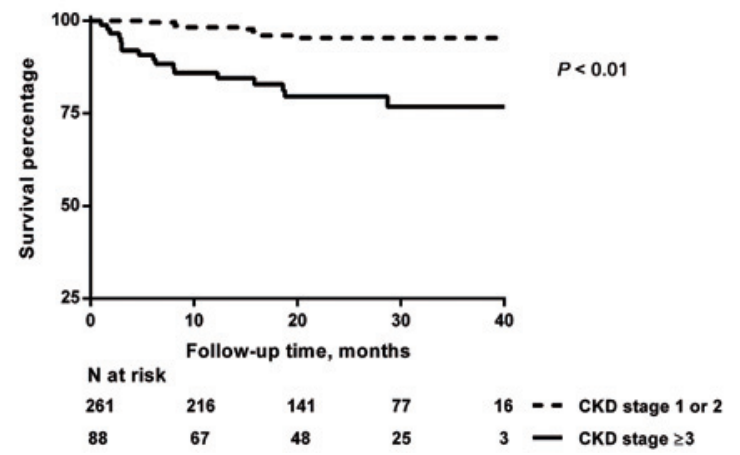

B

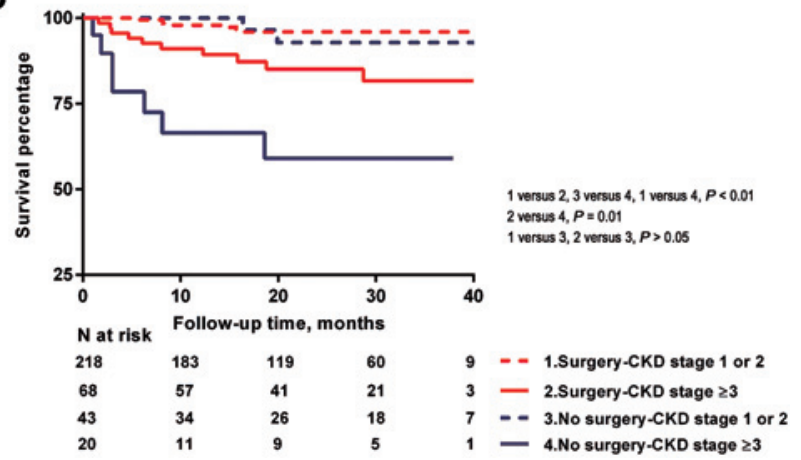

Figure 2. A: Kaplan-Meier analysis comparing incidence of mortality between chronic kidney disease (CKD) stage 1 or 2 and CKD stage $\geq 3$ in all patients. B: Kaplan-Meier analysis comparing incidence of mortality between CKD stage 1 or 2 and CKD stage $\geq 3$ in patients who underwent surgery (red color) and in those who did not (blue color).

Table III. Cox Regression Analysis of Valve Surgery in Association with Mortality

\begin{tabular}{lcccccc}
\hline & All patients & CKD stage $\geq 3$ & \multicolumn{2}{c}{ CKD stage 1 or 2} \\
& HR $(95 \%$ CI $)$ & $P$ & HR $(95 \%$ CI $)$ & $P$ & HR $(95 \%$ CI $)$ & $P$ \\
\hline Unadjusted & $0.37(0.17$ to 0.84$)$ & 0.02 & $0.32(0.12$ to 0.84$)$ & 0.02 & $0.66(0.14$ to 3.20$)$ & 0.61 \\
Adjusted* $^{*}$ & $0.45(0.19$ to 1.04$)$ & 0.06 & $0.32(0.12$ to 0.86$)$ & 0.02 & $0.87(0.17$ to 4.64$)$ & 0.87 \\
Adjusted $^{\&}$ & $0.39(0.16$ to 0.92$)$ & 0.03 & $0.33(0.12$ to 0.93$)$ & 0.04 & $0.91(0.14$ to 5.91$)$ & 0.92 \\
\hline
\end{tabular}

Adjusted*: adjusted age, sex. Adjusted ${ }^{\&}$ : adjusted age, sex, diabetes mellitus, hypertension, hyperlipidemia, smoking, atrial fibrillation, ACEI/ARB, and baseline eGFR. Abbreviations similar to those of Table I.

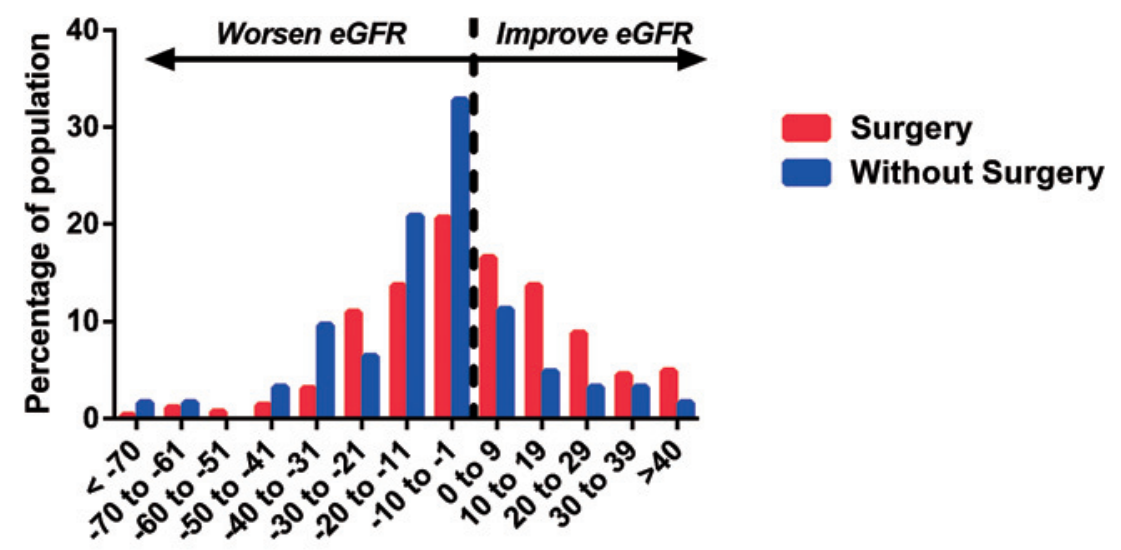

Percentage change in estimated GFR

Figure 3. Distribution of percentage change in estimated glomerular filtration rate (eGFR) in patients who underwent valvular surgery (red color) and in those who did not (blue color).

\section{Discussion}

The present study demonstrates that significant CKD is common in patients with significant VHD and increases with age. Importantly, valvular surgery in patients with concomitant significant CKD improved survival compared with continued medical therapy. The survival benefit of valvular surgery was, however, neutral in patients with no CKD. Furthermore, valvular surgery preserved renal function, whereas patients left untreated surgically had a significant decline in renal function.

Renal dysfunction is highly prevalent in patients with cardiovascular disease and has been reported to affect $25 \%$ to $56 \%$ of patients with chronic heart failure and $32 \%$ of patients with coronary artery disease. ${ }^{9-11)}$ The present study demonstrated that, compared with other cardiovascular diseases, renal dysfunction was present in $25 \%$ of patients with significant VHD. Furthermore, the presence of renal dysfunction was most likely to occur in elderly patients (up to $30 \%$ of the patients aged over 60 years). As the etiology of VHD now shifted toward degenerative from CRHD, patients presented with VHD will be more often elderly and thus the presence of concomitant CKD will be increasingly seen. ${ }^{1)}$ 
Table IV. Change in Renal Function in Patients with and without Valvular Surgery

\begin{tabular}{|c|c|c|c|c|c|c|}
\hline & \multicolumn{3}{|c|}{ eGFR decline $>30 \%$} & \multicolumn{3}{|c|}{ Annual rate of eGFR decline } \\
\hline & $n(\%)$ & OR $(95 \% \mathrm{CI})$ & $P$ & $\begin{array}{c}\text { Median } \\
\text { (Interquartile range) }\end{array}$ & $\mathrm{B}(95 \% \mathrm{CI})$ & $P$ \\
\hline Surgery $(n=286)$ & $19(6.6)$ & & 0.02 & $0.4(-6.2$ to 7.3$)$ & & $<0.01$ \\
\hline Without surgery $(n=63)$ & $10(15.9)$ & & & $-4.6(-23.0$ to 0$)$ & & \\
\hline \multicolumn{7}{|c|}{ Regression model (surgery versus without surgery) } \\
\hline Unadjusted & & $0.38(0.17$ to 0.86$)$ & 0.02 & & $-22.66(-34.74$ to -10.59$)$ & $<0.01$ \\
\hline Adjusted* & & $0.46(0.20$ to 1.09$)$ & 0.08 & & $-20.01(-32.25$ to -7.86$)$ & $<0.01$ \\
\hline Adjusted $\&$ & & $0.40(0.16$ to 0.98$)$ & 0.04 & & $-21.67(-33.98$ to -9.39$)$ & $<0.01$ \\
\hline
\end{tabular}

Adjusted*: adjusted age, sex. Adjusted\&: adjusted age, sex, diabetes mellitus, hypertension, hyperlipidemia, smoking, atrial fibrillation, ACEI/ ARB, and baseline eGFR. Abbreviation similar to Table I.

The prognostic value of CKD in patients who undergo valvular surgery has been extensively reported, ${ }^{5,6}$ and thus the assessment of eGFR has been implemented in risk-scoring systems such as EuroScore II and the Society of Thoracic Surgeons score to calculate the postoperative mortality risk. A significant number of patients have consequently been denied surgery because they were considered to be too high risk, partly due to renal dysfunction. In agreement with the Euro Heart Survey on VHD, surgery was denied to $33 \%$ of patients with severe aortic stenosis ${ }^{15}$ and $49 \%$ of patients with severe mitral regurgitation. ${ }^{16)}$ In addition to older age and impaired LV ejection fraction, comorbidities such as renal dysfunction were characteristic of patients who were denied surgery. In the present study, surgery was denied to 33 patients, of which over $30 \%$ had concomitant CKD and did not undergo surgery despite clinically indicated. These findings thus confirmed that renal dysfunction was common in patients who were denied surgery.

The survival benefit of valvular surgery in patients who are symptomatic or with LV remodeling is well proven. However, the survival benefit of surgery in patients with concomitant $\mathrm{CKD}$, a group that is considered having high post-operative risk, has not been evaluated. The present study confirms that patients with concomitant CKD had a high risk of adverse events following surgery. Importantly, those who had CKD but did not receive surgery experienced the highest mortality rate. Notably, compared to patients who were managed medically, valvular surgery provided significant survival benefit to those with CKD. Collectively, these findings imply that in patients with significant VHD who are referred for surgery, the presence of CKD should not be considered only a risk factor for post-operative complications but rather an indicator of mortality if only treated medically. In addition, up to $30 \%$ of patients with $\mathrm{CKD}$ died while waiting for surgery. This finding thus suggests that patients with significant valvular disease and concomitant CKD had an unstable cardiac condition and were at high risk of mortality. A timely surgery is thus indicated to those with CKD in order to prevent premature mortality during the waiting period. Furthermore, due to the short follow-up period (median 21 months) of the current study, the positive impact of valvular surgery may not be reflected in patients without CKD.

Although renal dysfunction is strongly associated with mortality, ${ }^{17)}$ worsening of eGFR remains common among patients with heart failure. ${ }^{18)}$ Studies have failed to demonstrate any effective treatment that can prevent the worsening of renal function. ${ }^{19,20)}$ The present study demonstrated that in patients with VHD, renal function was preserved in those who underwent surgery but rapidly declined in those who did not. This finding is further evidence that surgical correction of the valvular status is an effective means to prevent progression of renal dysfunction in patients with VHD. Importantly, the rapid decline in eGFR in those without surgery confirms the causative role of significant VHD in the development of renal dysfunction, and that medical treatment alone will not be sufficient to slow the progress.

Clinical implications: The optimal timing of valvular surgery relies on individual risk-benefit analysis, and the decision-making process is generally based upon clinical experience. While guidelines recommend the severity of symptoms and worsened echocardiography parameters as major indications for surgery, the role of renal dysfunction in this regard has not been addressed. ${ }^{2,3)}$ The present study demonstrated that renal function declines rapidly in patients with significant VHD, and once renal dysfunction has developed, mortality is alarmingly high if surgery is not performed. Based on these observations, it is recommended that renal function should be evaluated frequently in patients with significant VHD and renal dysfunction as well as considered an important indication for early valvular surgery.

Limitations: The present study did not systemically calculate the Society of Thoracic Surgeons (STS) score or EuroScore II, and did not perform hemodynamic measurements to assess pre-surgical cardiac output and central venous pressure. These measures should be elucidated by future studies. Additionally, the present study included patients referred for valvular surgery with various etiologies and surgical procedures, and the results should thus be verified in individual types of VHD. Furthermore, the present evaluation is a comparative effectiveness study and, therefore, a randomized trial would be required to control for all confounders. A larger sample size is required to determine the best cutoff value of renal function in association with mortality in order to better define the optimal timing of surgery. Finally, renal function was evaluated in the present study by eGFR; studies using biomarkers such as cystatin $\mathrm{C}$ and microalbuminuria are required to provide additional information on renal function. 


\section{Conclusions}

The present study demonstrates that renal dysfunction is common in patients with significant VHD. Patients with significant VHD that was not corrected surgically exhibited a rapid decline in renal function and mortality was high once renal dysfunction developed. Monitoring renal function and identifying patients with CKD is, therefore, recommended. The latter should be considered an important indication for early surgery in patients with VHD.

\section{Acknowledgments}

We thank the medical and nursing staff of the Division of Cardiology, Queen Mary Hospital, for the help and support during this study.

\section{Disclosures}

Conflicts of interest: This study was not supported by any funding and no author has a real or perceived conflict of interest.

\section{References}

1. Iung B, Vahanian A. Epidemiology of valvular heart disease in the adult. Nat Rev Cardiol 2011; 8: 162-72.

2. Joint Task Force on the Management of Valvular Heart Disease of the European Society of Cardiology (ESC), European Association for Cardio-Thoracic Surgery (EACTS), Vahanian A, et al. Guidelines on the management of valvular heart disease (version 2012). Eur Heart J 2012; 33: 2451-96.

3. Nishimura RA, Otto CM, Bonow RO, et al. 2014 AHA/ACC guideline for the management of patients with valvular heart disease: a report of the American College of Cardiology/American Heart Association Task Force on Practice Guidelines. J Am Coll Cardiol 2014; 63: e57-185.

4. Levey AS, Coresh J. Chronic kidney disease. Lancet 2012; 379: 165-80.

5. Lin CY, Tsai FC, Chen YC, et al. Correlation of preoperative renal insufficiency with mortality and morbidity after aortic valve replacement: A propensity score matching analysis. Medicine (Baltimore) 2016; 95: e2576.

6. Yamamoto M, Hayashida K, Mouillet G, et al. Prognostic value of chronic kidney disease after transcatheter aortic valve implantation. J Am Coll Cardiol 2013; 62: 869-77.

7. Yiu KH, Wong A, Pu L, et al. Prognostic value of preoperative right ventricular geometry and tricuspid valve tethering area in patients undergoing tricuspid annuloplasty. Circulation 2014;
129: 87-92.

8. Levey AS, Coresh J, Greene T, et al. Using standardized serum creatinine values in the modification of diet in renal disease study equation for estimating glomerular filtration rate. Ann Intern Med 2006; 145: 247-54.

9. McAlister FA, Ezekowitz J, Tonelli M, Armstrong PW. Renal insufficiency and heart failure: prognostic and therapeutic implications from a prospective cohort study. Circulation 2004; 109: 1004-9.

10. Hillege HL, Nitsch D, Pfeffer MA, et al. Renal function as a predictor of outcome in a broad spectrum of patients with heart failure. Circulation 2006; 113: 671-8.

11. Shepherd J, Kastelein JJ, Bittner V, et al. Intensive lipid lowering with atorvastatin in patients with coronary heart disease and chronic kidney disease: the TNT (Treating to New Targets) study. J Am Coll Cardiol 2008; 51: 1448-52.

12. Fu L, Zhou Q, Zhu W, et al. Do Implantable cardioverter defibrillators reduce mortality in patients with chronic kidney disease at all stages? Int Heart J 2017; 58: 371-7.

13. Levey AS, Inker LA, Matsushita K, et al. GFR decline as an end point for clinical trials in CKD: a scientific workshop sponsored by the National Kidney Foundation and the US Food and Drug Administration. Am J Kidney Dis 2014; 64: 821-35.

14. Lang RM, Bierig M, Devereux RB, et al. Recommendations for chamber quantification: a report from the American Society of Echocardiography's Guidelines and Standards Committee and the Chamber Quantification Writing Group, developed in conjunction with the European Association of Echocardiography, a branch of the European Society of Cardiology. J Am Soc Echocardiogr 2005; 18: 1440-63.

15. Iung B, Cachier A, Baron G, et al. Decision-making in elderly patients with severe aortic stenosis: why are so many denied surgery? Eur Heart J 2005; 26: 2714-20.

16. Mirabel M, Iung B, Baron $\mathrm{G}$, et al. What are the characteristics of patients with severe, symptomatic, mitral regurgitation who are denied surgery? Eur Heart J 2007; 28: 1358-65.

17. Imamura $T$, Kinugawa K. Prognostic impacts of hyponatremia, renal dysfunction, and high-dose diuretics during a 10-year study period in 4,087 Japanese heart failure patients. Int Heart J 2016; 57: 657-8.

18. Damman K, Jaarsma T, Voors AA, Navis G, Hillege HL, van Veldhuisen DJ. Both in- and out-hospital worsening of renal function predict outcome in patients with heart failure: results from the Coordinating Study Evaluating Outcome of Advising and Counseling in Heart Failure $(\mathrm{COACH})$. Eur J Heart Fail 2009; 11: 847-54.

19. Massie BM, O'Connor CM, Metra M, et al. Rolofylline, an adenosine A1-receptor antagonist, in acute heart failure. N Engl J Med 2010; 363: 1419-28.

20. Chen HH, Anstrom KJ, Givertz MM, et al. Low-dose dopamine or low-dose nesiritide in acute heart failure with renal dysfunction: the ROSE acute heart failure randomized trial. JAMA 2013; 310: 2533-43. 